Bull. Korean Math. Soc. 39 (2002), No. 1, pp. 97-111

\title{
POLYGON SHORTENING MAKES (MOST) QUADRILATERALS CIRCULAR
}

\author{
THIERRY JECKO AND JEAN-CHRISTOPHE LÉGER
}

\begin{abstract}
We show that an analog of the Gage-Grayson-Hamilton Theorem for curves moving according to their mean curvature holds for the motion of quadrilaterals according to their Menger curvature.
\end{abstract}

\section{Introduction}

The Gage-Grayson-Hamilton Theorem ([1], [2], and [3]) states that if $C^{0}: \mathbb{S}^{1} \rightarrow \mathbb{C}$ is a $\mathcal{C}^{2}$ Jordan curve in the plane then there exists a family $C: \mathbb{S}^{1} \times\left[0, T^{*}[\rightarrow \mathbb{C}\right.$ of smooth Jordan curves in the plane such that $C(\cdot, 0)=C^{0}(\cdot), C(\cdot, t)$ tends to a constant function as $t \rightarrow T^{*}$ and

$$
\frac{\partial C}{\partial t}=\frac{\partial^{2} C}{\partial s^{2}} \text {. }
$$

Moreover, if we rescale the curves so that the enclosed areas are equal to $\pi$, we have that the rescaled curves tend (for example in the Hausdorff sense) to a circle of radius 1 .

Notice that the right hand side of equation (1) is the curvature vector of the curve $C(t)$ ( $s$ is the arc-length parameter on the curve $C(t)$ : it is not independent with the variable $t$ ). A family of curves $C$ depending on time $t$ satisfying equation (1) is said to be moving by its mean curvature.

A question (among others) this theorem raises is whether one can find a discrete version of the motion by mean curvature which preserves this theorem: this should be a test for a measuring how good a discretization process for this motion is.

In this short note, we shall present a proposition for a possible good discretization and show that at least for quadrilaterals an analog of the Gage-Grayson-Hamilton Theorem exists.

Received October 16, 2000. Revised May 7, 2001.

2000 Mathematics Subject Classification: 34D05, 53A15, 53C44.

Key words and phrases: motion by Menger curvature, cocyclicality, discretization, motion by mean curvature. 
For an ordered triple $(x, y, z)$ of distinct points in $\mathbb{C}$, let us call the Menger curvature of the triple the number

$$
c(x, y, z)=\left(\frac{x-y}{\overline{x-y}}-\frac{z-y}{\overline{z-y}}\right) \frac{1}{x-z} .
$$

This quantity will be our analog for polygons of the usual curvature for smooth curves. Observe that this is a natural quantity to have a look at. The fact is that $c(x, y, z)$ is zero if and only if the points $x, y$ and $z$ are colinear, $|c(x, y, z)|$ is the inverse of the radius of the circumcircle of the triangle $x y z$ and $y+\frac{c}{|c|^{2}}$ is the center of that circumcircle. Observe as well that this quantity has already been used for finding discrete analogs of well known curvature related theorems for Jordan curves (see for instance the introduction of [5] and the references therein for a variant of the four-vertex Theorem). Another beautiful use of it is related to boundedness properties of singular integral operators on subsets of $\mathbb{C}$ ([4] for instance).

For an integer $n \geq 3$ let us call a $n$-gon a map $y: \mathbb{Z} / n \mathbb{Z} \rightarrow \mathbb{C}$ such that, for all $i \in \mathbb{Z} / n \mathbb{Z}$, the points $y_{i-1}, y_{i}, y_{i+1}$ are distinct and let us define its Menger curvature $c$ by

$$
c_{i}:=c\left(y_{i-1}, y_{i}, y_{i+1}\right) \quad \forall i \in \mathbb{Z} / n \mathbb{Z}
$$

Remark that we have

$$
\sum_{i \in \mathbb{Z} / n \mathbb{Z}}\left(y_{i-1}-y_{i+1}\right) c_{i}=0
$$

For an interval $I \subset \mathbb{R}$, we will say that the family of $n$-gons $y$ : $\mathbb{Z} / n \mathbb{Z} \times I \rightarrow \mathbb{C}$ is moving by its Menger curvature if it satisfies the following differential system

$$
\forall i \in \mathbb{Z} / n \mathbb{Z}, \quad y_{i}^{\prime}:=\frac{d y_{i}}{d t}=c\left(y_{i-1}, y_{i}, y_{i+1}\right)=c_{i} .
$$

This kind of motion shares many common features with the classical motion by mean curvature, the first being that it preserves the shape of polygons which are cocyclical or colinear (i.e., the vertices lie on a single circle or a single line).

Another common property is the one of length shrinking. For an $n$-gon $y$, put its length $L=\sum_{i \in \mathbb{Z} / n \mathbb{Z}}\left|y_{i+1}-y_{i}\right|$. We have then, for a family $y(t)$ of $n$-gons moving by their Menger curvature that $L(t)$ is a nonincreasing function (Lemma 1). Moreover, we have

$$
L^{\prime}=-\sum_{i \in \mathbb{Z} / n \mathbb{Z}} \frac{\left|c_{i}\right|^{2}+\left|c_{i+1}\right|^{2}}{2}\left|y_{i+1}-y_{i}\right|,
$$


a formula which should be compared with formula (5) in [1] used for the usual motion by mean curvature.

In this note we will be interested in the case $n=4$, that is the motion of quadrilaterals (4-gons). In this case, as was already noted for general $n$ 's, cocyclical polygons keep their shapes. It turns out that parallelograms keep their shapes in the motion as well.

Here is now a rough idea of the main result (cf. Theorem 1 and Proposition 2) of this note. If we are given an initial quadrilateral, the Cauchy-Lipschitz Theorem ensures the short-time existence and uniqueness of a family of quadrilaterals evolving by their Menger curvature and starting with this quadrilateral.

It turns out that for "most" starting quadrilaterals, the maximal interval of existence $\left[0, T^{*}\right.$ [ is bounded and, as $t \rightarrow T^{*}$, the family collapses to a point whereas the limiting shape is a cocyclical quadrilateral.

We would like to stress on the fact that given a quadrilateral to start with, we have a simple test (see section 4 ) to see if the limiting shape in the evolution is cocyclical: we do not put any restrictions (such as convexity or no self-crossing) on the type of quadrilaterals we are looking at. The evolution in the singular cases is also described: the limiting shape is then a parallelogram or a colinear quadrilateral.

In the general situation ( $n \geq 4)$, we are able to show that, if $n \neq 0$ modulo 4 , the only invariant shapes are the cocyclical or colinear ones whereas, if $n=0$ modulo 4 , there is another invariant shape, namely a parallelogram described $n / 4$ times (see Proposition 1).

Open Problems: We do not know what happens in the case $n>4$, except the previous description of invariant shapes, and this may be an interesting subject of further investigation.

Another path of investigation is to find out if the motion of polygons by Menger curvature really approximates the usual motion of curves by curvature and if so, in which sense.

\section{General facts and first result}

In this section, $y: \mathbb{Z} / n \mathbb{Z} \times I \rightarrow \mathbb{C}$ will be a family of $n$-gons evolving by their Menger curvature. We collect some general facts about such a family. Most proofs are elementary computations. 
LemMa 1. (i) For any triple $(u, v, w)$ of distinct points in $\mathbb{C}$, we have

$$
2 \operatorname{Re}(c(u, v, w) \overline{(u-v)})=|c(u, v, w)|^{2}|u-v|^{2} .
$$

(ii) For all $i \in \mathbb{Z} / n \mathbb{Z}$, all $t \in I$,

$$
\frac{d\left|y_{i+1}-y_{i}\right|}{d t}=-\frac{\left|c_{i}\right|^{2}+\left|c_{i+1}\right|^{2}}{2}\left|y_{i+1}-y_{i}\right| .
$$

In particular, (6) holds true.

(iii) If $n$ is even, there exist some constant $b_{0}>0$ such that for all $t \in I$,

$\left|y_{1}-y_{2}\right|\left|y_{3}-y_{4}\right| \cdots\left|y_{n-1}-y_{n}\right|=b_{0}\left|y_{n}-y_{1}\right|\left|y_{2}-y_{3}\right| \cdots\left|y_{n-2}-y_{n-1}\right|$.

Proof. For (i), we have, as $v+\frac{c}{|c|^{2}}$ is the center of the circle passing through $u, v$ and $w$ of radius $1 /|c|$, that

$$
\left|u-v-\frac{c}{|c|^{2}}\right|^{2}=\frac{1}{|c|^{2}} \text {. }
$$

It remains to expand the above formula to get (i).

For (ii), it is enough to compute the derivative of $\left|y_{i+1}-y_{i}\right|^{2}$ and apply (i) to the triples $\left(y_{i-1}, y_{i}, y_{i+1}\right)$ and $\left(y_{i}, y_{i+1}, y_{i+2}\right)$.

Finally, (iii) follows from

$$
\begin{aligned}
& -\frac{1}{2} \sum_{i \in \mathbb{Z} / n \mathbb{Z}}\left|c_{i}\right|^{2}=\frac{d}{d t} \ln \left|y_{1}-y_{2}\right|\left|y_{3}-y_{4}\right| \cdots\left|y_{n-1}-y_{n}\right| \\
& -\frac{1}{2} \sum_{i \in \mathbb{Z} / n \mathbb{Z}}\left|c_{i}\right|^{2}=\frac{d}{d t} \ln \left|y_{n}-y_{1}\right|\left|y_{2}-y_{3}\right| \cdots\left|y_{n-2}-y_{n-1}\right| .
\end{aligned}
$$

It is straightforward to prove the

LEMMA 2. (i) If at time $t_{0} \in I, y\left(t_{0}\right)$ is a polygon whose vertices lie on a single line, then for all $t \in I, y(t)=y\left(t_{0}\right)$.

(ii) If at time $t_{0} \in I, y\left(t_{0}\right)$ is a polygon whose vertices lie on a single circle with center $x_{0}$ and radius $R_{0}$ then for all $t \in I$, for all $i \in \mathbb{Z} / n \mathbb{Z}$,

$$
y_{i}(t)=x_{0}+\frac{\sqrt{R_{0}^{2}-2\left(t-t_{0}\right)}}{R_{0}}\left(y_{i}\left(t_{0}\right)-x_{0}\right) .
$$

Lemma 2 gives two invariant shapes under the evolution. Is there some other invariant shape? The following proposition answers this question. 
Proposition 1. Assume that the shape of $y\left(t_{0}\right)$ is preserved under the evolution, that is, there are two complex-valued, differentiable functions defined on $I, a$ and $b, a$ being non-vanishing, such that

$$
\forall t \in I, \quad \forall i \in \mathbb{Z} / n \mathbb{Z}, \quad y_{i}(t)=a(t) y_{i}\left(t_{0}\right)+b(t),
$$

then,

(i) $y\left(t_{0}\right)$ is a colinear or cocyclical polygon,

(ii) or $n=0$ modulo $4, y_{i+4}=y_{i}$ for all $i \in \mathbb{Z} / n \mathbb{Z}$, and $y_{1}, y_{2}, y_{3}, y_{4}$ form a parallelogram i.e., $y_{1}-y_{2}=y_{4}-y_{3}$.

Proof. Since the shape is preserved,

$$
0=\frac{d}{d t} \frac{\left|y_{i+1}-y_{i}\right|}{\left|y_{i+2}-y_{i+1}\right|} \text {. }
$$

Using Lemma 1, (ii), we get $\left|c_{i+2}(t)\right|=\left|c_{i}(t)\right|$. Now, using (2) and (5), we obtain, for any $i \in \mathbb{Z} / n \mathbb{Z}$,

$$
c_{i}(t)=\frac{d a}{d t}(t) y_{i}\left(t_{0}\right)+\frac{d b}{d t}(t) \text { and } c_{i}\left(t_{0}\right)=\bar{a}(t) c_{i}(t),
$$

so that, for any $i \in \mathbb{Z} / n \mathbb{Z}$,

$$
c_{i}\left(t_{0}\right)=\bar{a}(t) \frac{d a}{d t}(t) y_{i}\left(t_{0}\right)+\bar{a}(t) \frac{d b}{d t}(t) .
$$

From now on, we consider only the quadrilateral $y\left(t_{0}\right)$ so we drop the reference to the time $t_{0}$.

If $a$ is constant, the $c_{i}$ 's are equal. Assume they are nonzero. Denote the centers of the circumcircles by $z_{i}:=y_{i}+c_{i} /\left|c_{i}\right|^{2}$. Thus the $\left(z_{i}-y_{i}\right)^{\prime} \mathrm{s}$ are equal. Since $y_{i+1}$ lies on the circle centered on $z_{i}$ containing $y_{i}$ and $y_{i}$ lies on the circle centered on $z_{i+1}$ containing $y_{i+1}$, this implies $y_{i}=y_{i+1}$, which is excluded. Therefore the $c_{i}$ 's are equal to 0 , which means that the $y_{i}$ 's lie on the same line.

If $a$ is not constant, each $y_{i}$ is the image of $c_{i}$ by some $i$-independent similitude

$$
\forall i \in \mathbb{Z} / n \mathbb{Z}, \quad y_{i}=\alpha c_{i}+\beta
$$

for some complex numbers $\alpha \neq 0$ and $\beta$. Let us denote by $r_{0}$ (resp. $r_{1}$ ) the common modulus of the $c_{2 i}$ 's (resp. $c_{2 i+1}$ 's). If $r_{0}=r_{1}$ then $r_{0}>0$ and (7) shows that the $y_{i}$ 's lie on the same circle. Assume $r_{0} \neq r_{1}$. This implies that $n$ is even.

From (7) and (i) in Lemma 1 , we derive that, for all $i \in \mathbb{Z} / n \mathbb{Z}$,

$$
\begin{aligned}
2 \operatorname{Re}\left(c_{2 i} \overline{\alpha\left(\overline{\left.c_{2 i+1}-c_{2 i}\right)}\right)}\right. & =|\alpha|^{2} r_{0}^{2}\left|c_{2 i+1}-c_{2 i}\right|^{2}, \\
2 \operatorname{Re}\left(c_{2 i+1} \overline{\alpha\left(c_{2 i}-c_{2 i+1}\right)}\right) & =|\alpha|^{2} r_{1}^{2}\left|c_{2 i+1}-c_{2 i}\right|^{2} .
\end{aligned}
$$


On one hand, the sum $(8)+(9)$ leads to

$$
-2 \operatorname{Re}(\alpha)=\left(r_{0}^{2}+r_{1}^{2}\right)|\alpha|^{2}
$$

and on the other hand, the difference gives, thanks to (10),

$$
2 \sin (\theta) \sin \left(\theta_{2 i}\right)=\left(r_{0}^{2}-r_{1}^{2}\right)|\alpha| \cos \left(\theta_{2 i}\right),
$$

where $\alpha=|\alpha| \exp (i \theta)$ and $\left.\left.\theta_{i} \in\right]-\pi ; \pi\right]$ denotes a measure of the oriented angle $\left(c_{i}, 0, c_{i+1}\right)$. In the same way for the points $c_{2 i}$ and $c_{2 i-1}$, we obtain

$$
2 \sin (\theta) \sin \left(-\theta_{2 i-1}\right)=\left(r_{0}^{2}-r_{1}^{2}\right)|\alpha| \cos \left(-\theta_{2 i-1}\right) \text {. }
$$

Since $y_{2 i-1} \neq y_{2 i+1}, c_{2 i-1} \neq c_{2 i+1}$ by $(7)$ and $\theta_{2 i} \neq-\theta_{2 i-1}$ because $\left|c_{2 i-1}\right|=\left|c_{2 i+1}\right|$. The equations (11) and (12) say that $\theta_{2 i}$ and $-\theta_{2 i-1}$ satisfy an $i$-independent equation, which solutions are equal modulo $\pi$. This yields that $c_{2 i-1}$ and $c_{2 i+1}$ are symmetric w.r.t. the origin. Similarly $c_{2 i}$ and $c_{2 i+2}$ are also symmetric w.r.t. the origin. Therefore the $\theta_{2 i}$ 's (resp. $\theta_{2 i-1}$ 's) are equal. This means that the quadrilaterals $\left(c_{2 i-1}, c_{2 i}, c_{2 i+1}, c_{2 i+2}\right)$ form the same parallelogram. This is possible only for $n=0$ modulo 4 . Using (7) again, we get the result.

\section{Specific facts for quadrilaterals}

We now specialize to the case of quadrilaterals or 4-gons (i.e., $n=4$ ).

LEMMA 3. (i) There exists a constant $\Delta \in \mathbb{C}^{*}$ such that for all $t \in I, y_{4}-y_{2}=\Delta\left(y_{3}-y_{1}\right)$.

(ii) There exists a constant $b_{0}>0$ such that for all $t \in I$,

$$
\left|y_{1}-y_{2}\right|\left|y_{3}-y_{4}\right|=b_{0}\left|y_{1}-y_{4}\right|\left|y_{3}-y_{2}\right| \text {. }
$$

Proof. For (i), we just observe that (5) and (4) imply that

$$
y_{1}^{\prime}\left(y_{4}-y_{2}\right)+y_{2}^{\prime}\left(y_{1}-y_{3}\right)+y_{3}^{\prime}\left(y_{2}-y_{4}\right)+y_{4}^{\prime}\left(y_{3}-y_{1}\right)=0
$$

so that $\left(y_{3}-y_{1}\right)\left(y_{4}-y_{2}\right)^{\prime}-\left(y_{3}-y_{1}\right)^{\prime}\left(y_{4}-y_{2}\right)=0$.

(ii) is just a rewriting of (iii) of Lemma 1 .

Among the invariant shapes (Proposition 1), we have already described the evolution of two of them in the general case (see Lemma 2). Before treating the third one for $n=4$, we want to stress on the fact that the following lemma also gives the evolution of the periodic parallelogram (i.e., the form (ii) in Proposition 1) in the general case. 
LEMMA 4. If for $t_{0} \in I, y\left(t_{0}\right)$ is a nonflat parallelogram with center $x_{0}$ then for any $t \in I, y(t)$ is a similar parallelogram with center $x_{0}$ given by

$$
\left\{\begin{array}{l}
y_{1}(t)=x_{0}+\lambda\left(t-t_{0}\right) / 2 \\
y_{3}(t)=x_{0}-\lambda\left(t-t_{0}\right) / 2 \\
y_{2}(t)=x_{0}+\Delta \lambda\left(t-t_{0}\right) / 2 \\
y_{4}(t)=x_{0}-\Delta \lambda\left(t-t_{0}\right) / 2
\end{array}\right.
$$

Here, we have put

$$
\Delta=\frac{y_{4}-y_{2}}{y_{3}-y_{1}}\left(t_{0}\right) \notin\{-1 ; 1\}
$$

and

$$
\lambda(t)=\lambda(0) \sqrt{\frac{|\lambda(0)|^{2}-\alpha t}{|\lambda(0)|^{2}}} e^{-i \frac{\beta}{\alpha} \ln \left|\frac{|\lambda(0)|^{2}-\alpha t}{|\lambda(0)|^{2}}\right|},
$$

where

$$
\alpha=8 \frac{(\operatorname{Im} \Delta)^{2}\left(|\Delta|^{2}+1\right)}{|\Delta|^{2}|\Delta+1|^{2}|\Delta-1|^{2}} \quad \text { and } \quad \beta=4 \frac{(\operatorname{Im} \Delta)(\operatorname{Re} \Delta)\left(|\Delta|^{2}-1\right)}{|\Delta|^{2}|\Delta+1|^{2}|\Delta-1|^{2}}
$$

Proof. Because of the uniqueness in the Cauchy-Lipschitz Theorem, it suffices to check that (13) is a solution of the differential system (5).

What ought to be said about this formula is that a nonflat parallelogram such that $|\Delta| \neq 1$ (that is, not a rectangle) evolving according to its Menger curvature shrinks to its center in finite time and that, while shrinking, it rotates more and more rapidly around that point. This is a very strong contrast with the evolution of a cocyclical quadrilateral which shrinks to the center of the circle in finite time but does not rotate at all.

As we are interested in the property of being cocyclical, it is natural to introduce the cross-ratio of a quadrilateral $z$. It is defined by

$$
B_{z}=\frac{\left(z_{1}-z_{2}\right)\left(z_{3}-z_{4}\right)}{\left(z_{1}-z_{4}\right)\left(z_{3}-z_{2}\right)} .
$$

The quantity $B_{z}$ cannot be 0 or 1 because we supposed that the points $z_{i}$ are distinct. It is well known that the quadrilateral $z$ is cocyclical or colinear if and only if $B_{z}$ is a real number. An important feature of $B_{z}$ is that it is a scale and rotation invariant quantity: this is the quantity we will use to characterize cocyclical shapes. 
What is lesser known, although easy, is the following scale and rotation invariant characterization of parallelograms in terms of $\Delta_{z}=\frac{z_{4}-z_{2}}{z_{3}-z_{1}}$ and $B_{z}: z$ is a parallelogram if and only if

$$
B_{z}=\left(\frac{\Delta_{z}-1}{\Delta_{z}+1}\right)^{2}
$$

This is due to the following relation

$$
\left(\left(z_{2}+z_{4}\right)-\left(z_{1}+z_{3}\right)\right)^{2}=\frac{\left(z_{3}-z_{1}\right)^{2}}{1-B_{z}}\left(\left(\Delta_{z}-1\right)^{2}-B_{z}\left(\Delta_{z}+1\right)^{2}\right)
$$

Recall that $\Delta=\Delta_{y(t)}$ is constant during the motion and that $B(t)=$ $B_{y(t)}$ is such that $|B(t)|=b_{0}$ is constant as well (see Lemma 3). The next lemma states that we know quite precisely how $B$ moves on the circle with center 0 and radius $b_{0}$.

Lemma 5. (i) B satisfies the following differential equation

$$
B^{\prime}=\frac{\left|\left(y_{2}+y_{4}\right)-\left(y_{1}+y_{3}\right)\right|^{2}}{\left|y_{2}-y_{1}\right|^{2}\left|y_{4}-y_{3}\right|^{2}} B(B-\bar{B})
$$

(ii) Unless we are looking at the evolution of a fanily of parallelograms or of cocyclical or colinear quadrilaterals, Re $B$ is a decreasing function and unless we are looking at the evolution of a family of cocyclical or colinear quadrilaterals, $\operatorname{Im} B$ is of constant sign.

(iii) Put, for a fixed $t_{0} \in I$ and all $t \in I$,

$$
\psi(t)=\int_{t_{0}}^{t} \frac{\left|\left(y_{2}+y_{4}\right)-\left(y_{1}+y_{3}\right)\right|^{2}}{\left|y_{2}-y_{1}\right|^{2}\left|y_{4}-y_{3}\right|^{2}} d \tau
$$

Then there exists a constant $C$ such that for all $t \in I$,

$$
\operatorname{Re} B(t)=b_{0} \frac{1-C e^{4 b_{0} \psi(t)}}{1+C e^{4 b_{0} \psi(t)}}
$$

Proof. Equation (17) is a straightforward computation. Notice first that for any $i \in \mathbb{Z} / 4 \mathbb{Z}$,

$$
\frac{\partial B}{\partial y_{i}}=(-1)^{i+1} \frac{y_{i+1}-y_{i-1}}{\left(y_{i+1}-y_{i}\right)\left(y_{i-1}-y_{i}\right)} B,
$$


so that,

$$
\begin{aligned}
& B^{\prime}=\sum_{i \in \mathbb{Z} / 4 \dot{Z}} c_{i} \frac{\partial B}{\partial y_{i}}
\end{aligned}
$$

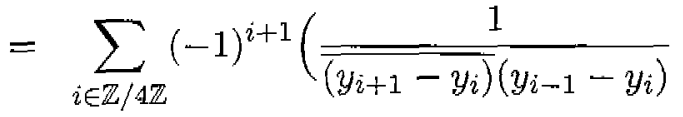

$$
\begin{aligned}
& \left.-\frac{1}{\left(y_{i+1}-y_{i}\right) \overline{\left(y_{i-1}-y_{i}\right)}}\right) B \\
& =2 \operatorname{im}\left[\frac{1}{\left|y_{2}-y_{1}\right|^{2}}\left(\frac{y_{2}-y_{1}}{y_{4}-y_{1}}-\frac{\overline{y_{2}-y_{1}}}{\overline{y_{2}-y_{3}}}\right)+\right. \\
& \left.+\frac{1}{\left|y_{4}-y_{3}\right|^{2}}\left(\frac{y_{4}-y_{3}}{y_{2}-y_{3}}-\frac{\overline{y_{4}-y_{3}}}{\overline{y_{4}-y_{1}}}\right)\right] B \\
& =2 \operatorname{im}\left[\frac{1}{\left|y_{2}-y_{1}\right|^{2}}\left(\frac{y_{2}-y_{3}}{y_{4}-y_{3}} B-\frac{\overline{y_{4}}-y_{1}}{\overline{y_{4}-y_{3}}} \bar{B}\right)+\right. \\
& \left.+\frac{1}{\left|y_{1}-y_{3}\right|^{2}}\left(\frac{y_{4}-y_{1}}{y_{2}-y_{1}} B-\frac{\overline{y_{2}-y_{3}}}{\overline{y_{2}-y_{1}}} \bar{B}\right)\right] B \\
& =2 \operatorname{imm}\left[\frac{1}{\left|y_{2}-y_{1}\right|^{2}}\left(\frac{y_{2}-y_{3}}{y_{4}-y_{3}} B+\frac{y_{4}-y_{1}}{y_{4}-y_{3}} B\right)+\right. \\
& \left.+\frac{1}{\left|y_{4}-y_{3}\right|^{2}}\left(\frac{y_{4}-y_{1}}{y_{2}-y_{1}} B+\frac{y_{2}-y_{3}}{y_{2}-y_{1}} B\right)\right] B \\
& =\frac{\left|\left(y_{2}+y_{4}\right)-\left(y_{1}+y_{3}\right)\right|^{2}}{\left|y_{2}-y_{1}\right|^{2}\left|y_{4}-y_{3}\right|^{2}} B(B-\bar{B}) \text {. }
\end{aligned}
$$

Let us call

$$
r(t)=\frac{\left|\left(y_{2}+y_{4}\right)-\left(y_{1}+y_{3}\right)\right|^{2}}{\left|y_{2}-y_{1}\right|^{2}\left|y_{4}-y_{3}\right|^{2}} .
$$

Equation (20) can be rewritten as

$$
\left\{\begin{array}{lll}
\operatorname{Re} B^{\prime} & = & -2 r(t)(\operatorname{Im} B)^{2} \\
\operatorname{Im} B^{\prime} & = & 2 r(t)(\operatorname{Im} B)(\operatorname{Re} B)
\end{array}=-2 r(t)\left(b_{0}^{2}-(\operatorname{Re} B)^{2}\right)\right.
$$

We know that $B$ moves on a circle (Lemma 3 ) and that unless we are looking at a cocyclical or colinear evolving family, because of uniqueness in the Cauchy-Lipschitz Theorem, $B$ cannot be real (Lemma 2) so that $\operatorname{Im} B$ cannot be zero. As it is continuous on the interval $I$, it is of constant sign. Now we observe that $r(t)$ vanishes if and only if $y(t)$ is a parallelogram: this can only happen if we are looking at an evolving family of parallelograms (Lemma 4). Hence, we have (ii). (iii) follows by solving the differential equation governing $\operatorname{Re} B$. 


\section{The main results}

Let us recall that for a quadrilateral $y^{0}$, we put $\Delta^{0}=\left(y_{4}^{0}-y_{2}^{0}\right) /\left(y_{3}^{0}-\right.$ $\left.y_{1}^{0}\right)$. Observe that we can suppose $\Delta^{0} \neq-1$ because if this was the case we could swap $y_{2}$ and $y_{4}$ without changing the geometry of the problem. Let us put $B^{0}=B_{y^{\circ}}$ defined by (14).

We will say that $y^{0}$ is of type (0) if $y^{0}$ is a parallelogram or if $y^{0}$ is cocyclical or colinear. Observe that Lemmas 2 and 4 tell us how such quadrilaterals evolve.

We will say that $y^{0}$ is of type (I) if $\left|B^{0}\right|=\left|\frac{\Delta^{0}-1}{\Delta^{0}+1}\right|^{2}$.

$$
\operatorname{Im} B^{0} \cdot \operatorname{Im}\left(\frac{\Delta^{0}-1}{\Delta^{0}+1}\right)^{2}>0 \text { and } \operatorname{Re} B^{0}>\operatorname{Re}\left(\frac{\Delta^{0}-1}{\Delta^{0}+1}\right)^{2}
$$

We will say that $y^{0}$ is of type (II) in the other cases. (See Figure 1.)

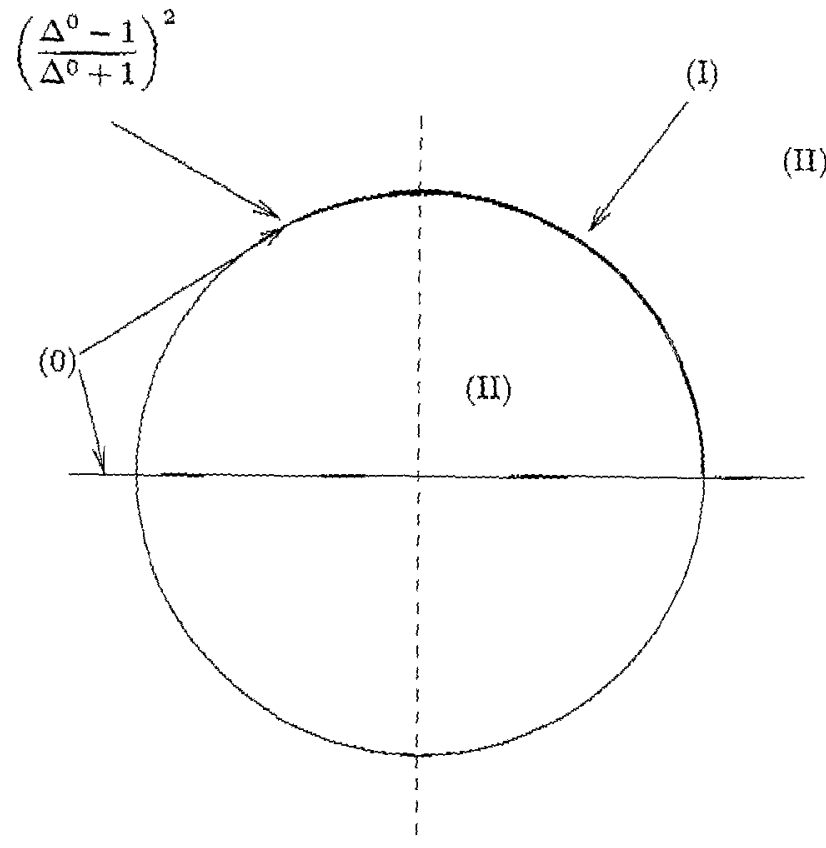

Figure 1. If $B^{0}$ is on the arc (I), the quadrilateral is of type (I), if $B^{0}$ is in zone (II), the quadrilateral is of type (II). 
THEOREM 1. Let $y^{0}$ be a quadrilateral then there exists an interval $I=\left[0, T^{*}\right.$ [and a unique family $y$ of quadrilaterals defined on $I$, evolving by Menger curvature such that $y(0)=y^{0}$. Moreover,

- If $y^{0}$ is of type (I) then as $t \rightarrow T^{*}$,

$$
B(t) \rightarrow\left(\frac{\Delta^{0}-1}{\Delta^{0}+1}\right)^{2}
$$

and, the rescaled quadrilaterals $\frac{y-\left(y_{3}+y_{1}\right) / 2}{y_{3}-y_{1}}$ tend to the parallelogram

$$
\left(-\frac{1}{2},-\frac{\Delta^{0}}{2},+\frac{1}{2},+\frac{\Delta^{0}}{2}\right) \text {. }
$$

- If $y^{0}$ is of type (II) then as $t \rightarrow T^{*}$,

$$
B(t) \rightarrow-\left|B^{0}\right|
$$

and, the rescaled quadrilaterals $\frac{y-\left(y_{3}+y_{1}\right) / 2}{y_{3}-y_{1}}$ tend to the cocyclical or colinear quadrilateral

$$
\left(-\frac{1}{2}, C^{0}-\frac{\Delta^{0}}{2},+\frac{1}{2}, C^{0}+\frac{\Delta^{0}}{2}\right)
$$

where

$$
C^{0}=\frac{1}{2} \sqrt{\frac{\left(\Delta^{0}-1\right)^{2}+\left|B^{0}\right|\left(\Delta^{0}+1\right)^{2}}{1+\left|B^{0}\right|}}
$$

and $\sqrt{ }$ is the analytic continuation of the square root along the arc described by $\left.\left[\left(\Delta^{0}-1\right)^{2}-B\left(\Delta^{0}+1\right)^{2}\right)\right] /(1-B)$ as $B$ describes the small arc between $B^{0}$ and $-\left|B^{0}\right|$ and such that

$$
\frac{\left(y_{4}^{0}+y_{2}^{0}\right)-\left(y_{3}^{0}+y_{1}^{0}\right)}{2\left(y_{3}^{0}-y_{1}^{0}\right)}=\frac{1}{2} \sqrt{\frac{\left(\Delta^{0}-1\right)^{2}-B^{0}\left(\Delta^{0}+1\right)^{2}}{1-B^{0}}} .
$$

Proof. Let us suppose that $y^{0}$ is not of type (0) and let us use the Cauchy-Lipschitz Theorem to get an evolving family starting at $y^{0}$ which is a maximal solution, defined on the interval $\left[0, T^{*}[\right.$, of the differential system (5) with initial condition $y(0)=y^{0}$.

By Lemma 5, we know that $B(t)$ has a limit $B_{T^{*}}$ as $t \rightarrow T^{*}$. To get the Theorem, we only have to prove that $B_{T^{*}}=-\left|B^{0}\right|$ or $B_{T^{*}}=$ $\left(\left(\Delta^{0}-1\right) /\left(\Delta^{0}+1\right)\right)^{2}$.

The statements about the rescaled quadrilaterals will be just an easy use of (16) to find the limit of the midpoint of the diagonals of the rescaled quadrilateral and the fact that $\Delta$ is constant. 
Observe also that, because of the way $B$ moves on the circle of center 0 and radius $\left|B^{0}\right|$, if $y^{0}$ is of type (I) then $B_{T^{*}}$ cannot be $-\left|B^{0}\right|$ and if $y^{0}$ is of type (II) then $B_{T^{*}}$ cannot be $\left(\left(\Delta^{0}-1\right) /\left(\Delta^{0}+1\right)\right)^{2}$.

Let us suppose that

$$
B_{T^{*}} \neq\left(\frac{\Delta^{0}-1}{\Delta^{0}+1}\right)^{2}
$$

then because of (16) and an easy compactness argument, there exists a real constant $K \geq 1$ such that for all $t \in\left[0, T^{*}[\right.$,

$$
K^{-1}\left|y_{3}-y_{1}\right| \leq\left|\left(y_{2}+y_{4}\right)-\left(y_{1}+y_{3}\right)\right| \leq K\left|y_{3}-y_{1}\right| \text {. }
$$

Hence, because of the triangle inequality, we have that for any $i \in \mathbb{Z} / 4 \mathbb{Z}$,

$$
\left|y_{i+1}-y_{i}\right| \leq \frac{1}{2}(1+K+|\Delta|)\left|y_{3}-y_{1}\right|
$$

Hence,

$$
L(t) \leq 2(1+K+|\Delta|)\left|y_{3}-y_{1}\right|
$$

Moreover, using (6), Lemma 3 , and

$$
|c(u, v, w)| \leq \frac{2}{|u-w|}
$$

for any distinct points $(u, v, w)$ in $\mathbb{C}$, we obtain that, for all $t \in\left[0, T^{*}[\right.$,

$$
\begin{aligned}
-L^{\prime}(t) & =\frac{1}{2} \sum_{i \in \mathbb{Z} / 4 \mathbb{Z}}\left(\left|c_{i+1}\right|^{2}+\left|c_{i}\right|^{2}\right)\left|y_{i+1}-y_{i}\right| \\
& \leq K^{\prime}\left|y_{3}-y_{1}\right|^{-1}
\end{aligned}
$$

This gives us that the function $\psi$ defined in (18) satisfies for all $t \in$ $\left[0, T^{*}[\right.$, on one hand, by $(21),(22)$, and (23),

$$
\begin{aligned}
\psi(t) & =\int_{0}^{t} \frac{\left|\left(y_{2}+y_{4}\right)-\left(y_{1}+y_{3}\right)\right|^{2}}{\left|y_{2}-y_{1}\right|^{2}\left|y_{4}-y_{3}\right|^{2}} d \tau \\
& \geq K^{\prime \prime} \int_{0}^{t} \frac{d \tau}{L^{2}(\tau)}
\end{aligned}
$$

and, on the other hand, by (21), (22), and (25),

$$
\begin{aligned}
\psi(t) & \geq K^{\prime \prime \prime} \int_{0}^{t} \frac{-L^{\prime}(\tau) d \tau}{L(\tau)} \\
& \geq K^{\prime \prime \prime} \ln \frac{L(0)}{L(t)}
\end{aligned}
$$


Because of Lemma 5 , in order to have $B(t) \rightarrow-\left|B^{0}\right|$ as $t \rightarrow T^{*}$, it remains to show that $\psi(t) \rightarrow \infty$ as $t \rightarrow T^{*}$.

There are two possibilities, either $T^{*}=+\infty$ or $T^{*}<+\infty$.

If $T^{*}=+\infty$ then as $\frac{1}{L^{2}}$ is nondecreasing, inequality (26) gives us the result.

If $T^{*}<+\infty$ and $L(t) \nrightarrow 0$ as $t \rightarrow T^{*}$ then we claim that $y(t) \rightarrow y\left(T^{*}\right)$ where $y\left(T^{*}\right)$ is a quadrilateral. The Cauchy-Lipschitz Theorem allows us to extend the evolution beyond $T^{*}$ thus contradicting the maximality of the family. As $L(t) \rightarrow 0$, inequality (27) shows that $\psi(t) \rightarrow \infty$ so that we get the result.

It remains to show why $y(t)$ tends to some quadrilateral as $t \rightarrow T^{*}$ if $L(t) \nrightarrow 0$. The lengths of the edges $\left|y_{i+1}-y_{i}\right|$ are nonincreasing (Lemma 1) and positive functions hence they have limits $l_{i}$. Because of Lemma 3 , the numbers $l_{i}$ are related by the relation

$$
l_{1} l_{3}=\left|B^{0}\right| l_{2} l_{4} \text {. }
$$

None of the numbers $l_{i}$ can be zero. If, for example $l_{1}=0$ then either $l_{2}$ or $l_{4}$ is zero. Suppose it is $l_{2}$ then we would have by (23)

$$
\begin{aligned}
L(t) & \leq K^{\prime}\left|y_{1}-y_{3}\right| \\
& \leq K^{\prime}\left(\left|y_{1}-y_{2}\right|+\left|y_{2}-y_{3}\right|\right) \\
& \rightarrow K^{\prime}\left(l_{1}+l_{2}\right)=0,
\end{aligned}
$$

and this is a contradiction with $L(t) \neq 0$. Now we have that there exists a constant $l_{0}>0$ such that for all $i \in \mathbb{Z} / 4 \mathbb{Z}$, for all $t \in\left[0, T^{*}[\right.$,

$$
\left|y_{i+1}-y_{i}\right|>l_{0} \text {. }
$$

Thanks to (22), there exists some other constant $l>0$ such that, for all $t \in\left[0, T^{*}[\right.$,

$$
\left|y_{3}-y_{1}\right|>l \text { and }\left|y_{4}-y_{2}\right|>l .
$$

This shows, using (24), that for all $i \in \mathbb{Z} / 4 \mathbb{Z}$, for all $t \in\left[0, T^{*}\right.$,

$$
\left|c_{i}\right|<\frac{2}{l}
$$

Coming back to the differential system (5), this shows that the functions $y_{i}$ are $\frac{2}{l}$-Lipschitz on $\left[0, T^{*}\right.$. Hence they have limits $y_{i}\left(T^{*}\right)$ as $t \rightarrow T^{*}$ and the map $i \mapsto y_{i}\left(T^{*}\right)$ is injective by (28) and (29).

We have seen that if we start with a colinear quadrilateral $y^{0}$ then $T^{*}=\infty$ whereas if $y^{0}$ is a cocyclical quadrilateral or a parallelogram then $T^{*}$ is finite (see Lemma 2 and 4 ). We can observe that the only 
way to have a colinear shape in the limit is to have $\Delta^{0}$ real. The following proposition shows that the situation when $\Delta^{0}$ is not real is well understood.

Proposition 2. If $\Delta^{0} \notin \mathbb{R}$ then $T^{*}<\infty, T^{*}=C . L(0)^{2}$ where $C$ is a constant depending only on the shape of $y^{0}$.

Moreover, as $t \rightarrow T^{*}, L(t) \rightarrow 0$ and there exists a point $x \in \mathbb{C}$ such that $y(t) \rightarrow(x, x, x, x)$.

Proof. Theorem 1 shows that if $\Delta^{0} \notin \mathbb{R}$, the rescaled quadrilaterals $\left(y-\left(y_{3}+y_{1}\right) / 2\right) /\left(y_{3}-y_{1}\right)$ tend to a noncolinear quadrilateral. Hence there exists $0<t_{0}<T^{*}$ such that for $t_{0}<t<T^{*}$, the quantities $\left|y_{3}-y_{1}\right|,\left|y_{i+1}-y_{i}\right|, 1 /\left|c_{i}\right|$ are comparable within positive multiplicative constants. Because of Lemma 1, we have, for any $t \in\left[t_{0}, T^{*}[\right.$,

$$
\frac{d}{d t}\left|y_{2}-y_{1}\right| \leq \frac{-\epsilon}{\left|y_{2}-y_{1}\right|}
$$

where $\epsilon$ is a positive constant. Integrating, we get that

$$
t<\frac{\left|y_{2}\left(t_{0}\right)-y_{1}\left(t_{0}\right)\right|^{2}}{\epsilon}, \quad \text { hence } \quad T^{*} \leq \frac{\left|y_{2}\left(t_{0}\right)-y_{1}\left(t_{0}\right)\right|^{2}}{\epsilon} .
$$

Starting with $y^{0}$ of length $L^{0}$ and rescaling we get a quadrilateral $\tilde{y}^{0}$ of length 1. A simple homogeneity argument gives us that $T^{*}=\left(L^{0}\right)^{2} \tilde{T}^{*}$.

Just as in the proof of Theorem 1 , if $L(t) \nrightarrow 0$ as $t \rightarrow T^{*}$, we could contradict the maximality of $T^{*}$ by extending the motion beyond $T^{*}$.

Now, let us observe that by Theorem 1 and Lemma 1 , there exists a time $0<t_{0}<T^{*}$ and a constant $A>0$ such that for each $i \in \mathbb{Z} / 4 \mathbb{Z}$, for each $t_{0}<t<T^{*}$,

$$
\left|y_{i}^{\prime}\right| \leq-A L^{\prime}
$$

Therefore the functions $y_{i}^{\prime}$ are integrable over $\left[0, T^{*}\right.$ [ so that the functions $y_{i}$ have limits $y_{i}\left(T^{*}\right)$ as $t \rightarrow T^{*}$, which are equal because $L(t) \rightarrow 0$.

\section{References}

[1] M. Gage, Curve shortening makes convex curves circular, Invent. Math. 76 (1984), 357-364.

[2] M. Gage and R.S. Hamilton, The heat equation shrinking convex plane curves, J. Differential Geometry 26 (1986), 69-96.

[3] M. A. Grayson, The heat equation shrinks embeded plane curves to round points, J. Differential Geometry 26 (1987), 285-314.

[4] M. S. Melnikov and P. Mattila, and J. Verdera, The Cauchy integral, analytic capacity, and uniform rectifiablity, Annals of Math. 144 (1996), 127*136. 
[5] V. D. Sedykh, Discrete versions of the four-vertex theorem, In A. (ed.) et al. Khovanskij, editor, Topics in singularity theory. V.I. Arnold's 60th anniversary collection, volume 180 (34) of Transl., Ser. 2, Am. Math. Soc., pages 197-207. Providence, RI: American Mathematical Society, 1997.

ThIerry Jecko, IMR, UnIVERstté de Rennes I, CAMpus BeAUlieu, F-35042 RENNES CÉDEX, FranCE

E-mail: jecko@maths.univ-rennes1.fr

JEAN-Christophe LÉGer, DÉPaRTEMENT de Mathématiques, BÂt. 425, Uñversité de Paris Sud, F-91405 Orsay CÉdex, France

E-mail: Jean-Christophe.Leger@math.u-psud.fr 